\title{
Something as simple and as complex as fibrocystic masthopathy review of $M R I$ findings in a case report
}

\begin{abstract}
Purpose: conduct a review of the findings on MRI of fibrocystic breast disease, taking into account existing short bibliography about it, from a complex case registered in our service.

Discussion: A large part of both clinical and subclinical lesions found in our daily work are benign and correspond with fibrocystic changes (FCC). This entity is complex and part exhibit traits that define characteristically in MRI and diagnose correctly, but there is another small group of changes that can take the image aspect of cancer. From a complex case, we review the main aspects of the FCC in MRI face to better understand a very common condition, although, but at the same time unknown in MRI technique.

Conclusion: more than $50 \%$ of lesions we see in the context of FCC show morphological and kinetic criteria of malignancy, so in the present moment we cannot avoid biopsies. The most relevant features in MRI in the FCC is non-mass regional enhancement and predominantly curves type II.
\end{abstract}

Keywords: breast, MRI, mastopathy, fibrocystic change, FCC, predominantly, lump
Volume 2 Issue I - 2017

\section{Estela Fernadez}

Hospital Begoa, Spain

\begin{abstract}
Correspondence: Estela Fernadez, Hospital Begoa,AVDA. Pablo Iglesias, NÂ 92. 33204, Spain, Tel +34 6067424 73, Email estelacuadriello@gmail.com
\end{abstract}

Received: September 25, 2016 | Published: January 23, 2017

\section{Review}

This is the case of 39years old woman, with no family history of breast cancer, with painless palpable lump recently appeared on CSI left breast, without blushing or heat. The patient comes to our Unit to start imaging studies. Mammographic, ultrasonographic and MR breast findings, and anatomo-pathological analysis are described below. In the mammography we can see a left global asymmetry that primarily affects upper quadrants (Figure 1), associating faint and few fine and heterogeneous scattered microcalcifications. Sonographically a mass measuring $47 \mathrm{~mm}$, consisting of numerous small and simple cysts grouped, with some ectatic canalicular segments and moderate hyperemia regional (Figure 2) with a hard and stiff behavior in the US-elastography, so additional histological study is made.
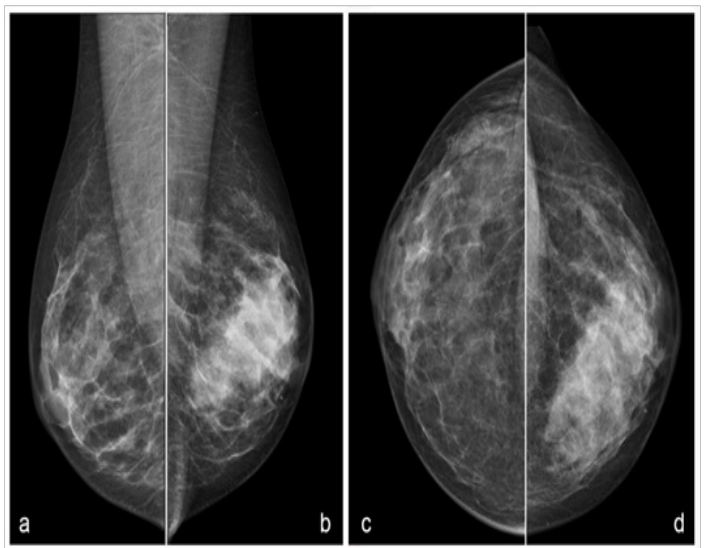

Figure I Left global asymmetry seen in CC and OML views, corresponding with palpable mass.

$14 \mathrm{G}$ biopsy is performed using ultrasound with the pathologic result of "fibrocystic changes". Given these findings it was decided to perform an image control in 3 months, in this case with breast MRI, to assess behavior and evolution of the lesion, in which we found an asymmetric regional enhancement in the upper left quadrants, with heterogeneous internal enhancement with wash-out curves (Figure 3 ) and decrease of ADC value. Therefore, we classify it as BIRADS 5 recommending second look ultrasound and new biopsy. In this case, we use $11 \mathrm{G}$ needle, extracting 6 cylinders with histopathologic result of "complex fibrocystic breast disease with intraductal microcalcifications". In posterior clinical session it determined make a lumpectomy to remove the lesion for a complete histological study.
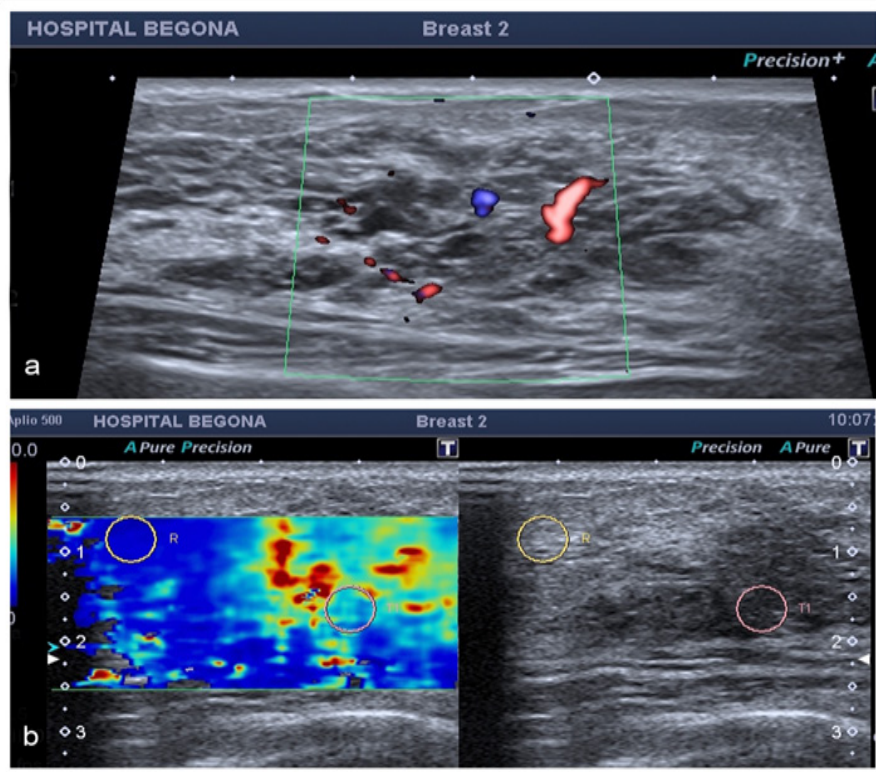

Figure 2 We detect a hipoechoic mass on sonography examination fundamentally composed of cysts and dilated ductal segments, with vessels and stiff areas within it in elastographic study which we made with an Applio 500, Toshiba medical Systems. 

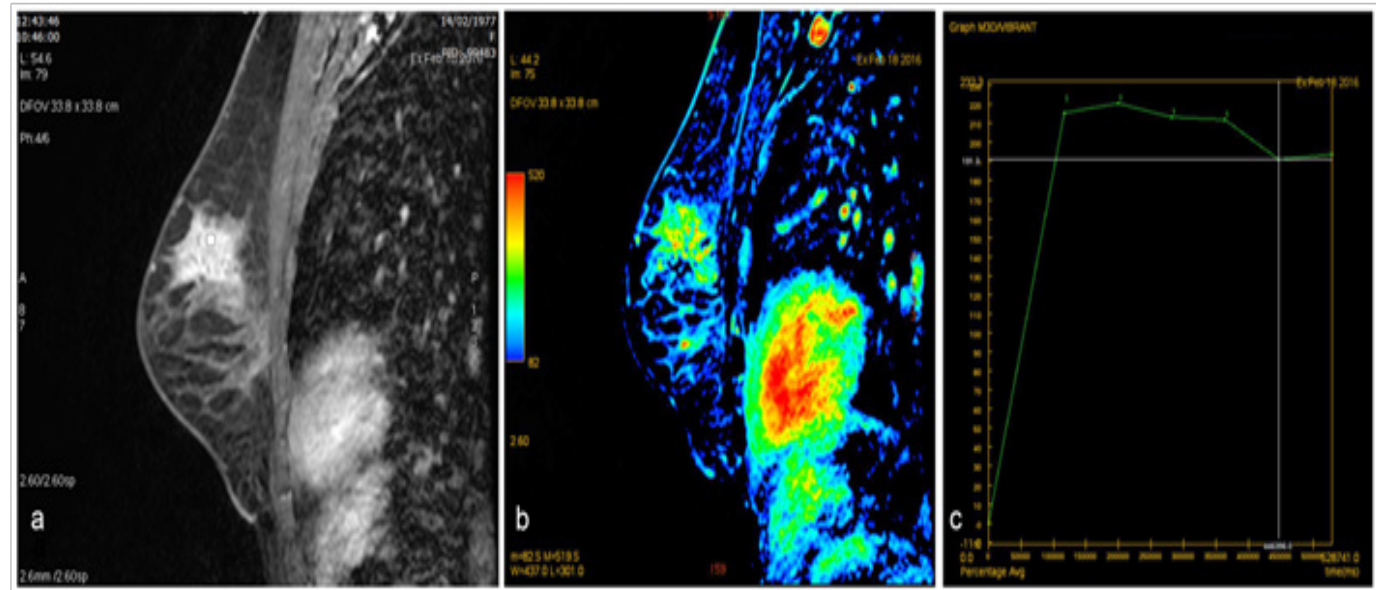

Figure 3 There is an important asymmetric regional enhancement in the upper left quadrants in contrast-enhanced TI-weighted fat-saturated

A. With internal heterogeneus enhancement.

B. Color-coded map shows foci with the maximum slope of enhancement increase (red) after contrast material injection.

C. Wash-out curve or type III.

Histological result of the piece finally threw a "extensive papillomatosis with intraductal apocrine changes and focal epithelial hyperplasia with atypia and complex fibrocystic breast disease with sclerosing adenosis". P-63 immunostaining performed, and CK-Actin m.liso 34B12 myoepithelial cells clearly observing and confirming the diagnosis of fibrocystic breast disease complex".

This case highlights the complexity of a benign entity, known to all, debuting as a false positive MRI at the expense of severe proliferative change. This is a benign process with radiological border-line and suspicious presentations, that shares morphology and kinetics features of malignancy on MR. Therefore we need to make a small literature review of a so simple entity and so complex as the same time, as the mammary dysplasia, mainly from the point of view of MRI, given the short literature exists about it.

Among all benign disease that affects the breast at some period of life of women, fibrocystic breast disease is the most anxiety and anguish generated, being one of the most frequent reasons for consultation, estimating that between 45 and $85 \%$ of patients consult for this reason. ${ }^{1}$

The nomenclature to refer to this entity is comprehensive: fibrocystic breast disease, fibrocystic changes, mammary dysplasia, scleral-cystic breast disease, fibrocystic breast changes or adenosis, mainly. Macroscopically it characterized by a marked proliferation of connective tissue and cysts of varying size, whose content is serousyellowish; microscopically they show epithelial lining these cysts that may have some degree of hyperplasia, sometimes adding an accessory layer of myoepithelial cells. ${ }^{2}$ Fibrocystic changes is the most common benign breast entity, in fact around $50 \%$ of women have these changes after autopsies that can be qualified of this nature. ${ }^{3,4}$

These changes are the result of the distortion produced periodically by menstrual changes on the ductal epithelium, and also secondary to changes in hormone levels of estrogen and progesterone, with the first increase and the second decrease in breast stroma. It is therefore a chronic and bilateral clinicopathological process consisting essentially of a proliferation of the epithelium lining the ducts, secondarily leading to a reaction fibrotic local connective component, which leads to the formation of cysts localized or diffuse way. ${ }^{1}$ It is a hyperplastic and proliferative process we observe, in particular, between 25 and 45 years old.

Fibrocystic tissue is often difficult to distinguish from normal breast parenchyma, acquiring a variable appearance depending on the water content and collagen of different tissues. ${ }^{5}$ In fact, the MR presentation can be very similar to mammographic, with small well defined and grouped cysts, or we can see after administration of contrast a dotted striking patchy pattern and diffuse, even as in our case, a regional enhancement or even a heterogeneous mass. ${ }^{6}$ Proliferative changes may also be arranged as a mass effect by the compressive effect on the stroma, altering the configuration and arrangement of the glandular component, which is indistinguishable from DCIS or invasive carcinoma. ${ }^{6}$

FCC is a benign and very common entity in our daily practice, with majority premenopausal presentation. ${ }^{7-9}$ Clinically it is seen in approximately $50 \%$ of women seeking consultation ${ }^{10}$ and about $90 \%$ of histological studies. ${ }^{7,11}$

This condition can cause pain in a particular area of the breast, in all of it or both, usually cyclically coinciding particularly with premenstrual changes (mastodinia). In the physic examination we can detect hardened areas or nodular areas that are located more frequently in CCSSEE. This behavior can easily simulate or hinder cancer diagnosis, so is always necessary imaging studies to exclude neoplasm's.

Mammography and ultrasound form the mainstay in diagnostic imaging in breast disease, because normally the breasts are dense and the mammographic sensitivity is limited. The complete parenchymal ultrasound study confirms the nature of nodular formations and characterizes those areas detected at palpable exploration. However, in a not inconsiderable percentage of cases of greater complexity it is necessary to complete the assessment with breast MRI to evaluate their behavior and exclude malignancy. This tool has gradually tightened his grip on diagnostic imaging of breast disease, and although it has taken years to standardize their indications, this is a sensitive and specific for the diagnosis of benign pathology technique. 


\section{MRI features}

The dynamic study in FCC will show regularly punctuate enhancements, bilateral distribution and generally patchy and diffuse, moderately symmetrical distribution. The numerous punctuate images we see usually have demonstrated respond to a focal adenosis. ${ }^{12}$

It is difficult to differentiate with this technique normal of fibrocystic tissue glandular parenchyma on $\mathrm{T} 1$ and $\mathrm{T} 2$ weighted sequences without contrast. The heterogeneity observed after administration of contrast is due in many cases to areas of epithelial proliferation alternating with areas of regressive changes, as fibrosis and cysts. True cystic disease occurs more frequently in the early 40 s, due to ductal epithelium hyperplasia and dilation of the ducts in the menstrual phase, not compensated by the involution of the parenchyma.

The FCC with epithelial proliferation has been associated with an increased risk of cancer, especially if it is accompanied by cellular Atypia. ${ }^{10,13,14}$ In fact, the main problem of patients affected by FCC is correctly diagnosed epithelial hyperplasia and malignant disease associated in many cases. Although these patients may express a clinical and image findings fairly typical, for some of them it is not enough only imaging study with mammography and ultrasound. Both malignant and benign breast conditions can share morphologic and kinetic patterns. Since MRI is increasingly used to screen young high risk women, diagnostic ambiguity will become more common due to the high incidence of fibrocystic breast seen in this age group. Benign breast disease includes proliferative changes, intraductal papillomas, granular cell tumors, fibroadenomas, mastitis, fat necrosis, pseudoangiomatous stromal hiperplasia, epidermal inclusion cyst, and benign intramammary lymph nodes. ${ }^{13,15}$

It's very important understand MR imaging features of FCC to exclude malignancy, avoid unnecessary biopsy, and of course, reduce patient anxiety. ${ }^{15}$ There are very few MRI studies about fibrocystic breast, and I consider relevant learn more about the behavior of this disease in MR given the ability to simulate and mask the cancer.

As we know the behavior of cancer in MRI it is studied from morphological and kinetic criteria, expressed by usual irregular or ill-defined morphologies and rapid initial uptake of contrast with subsequent washing. On the other hand, we also know that in many cases benign pathology may present criteria of malignancy in MRI, resulting in numerous biopsies and also in false positives. FCC has an indistinct vessel supply to the duct and can cause little inflammation around the duct. This can difficult the evaluation in MRI. But the other one displayed atypical epithelial hyperplasia accompanied with FCC. ${ }^{13,16}$ Kiyak et al. ${ }^{13}$ found that the analysis of enhancement kinetics may be more useful to identify premalignant lesions as atypical epithelial hyperplasia and cancer from other breast lesions provided that corrections are made for the true phase of menstrual cycle.

The enhancement is due to a number of biologic factors, including an increase in vessel number and size, increased vascular permeability, and increased cellularity. ${ }^{17,18}$ Maurice et al. ${ }^{19-21}$ reported that morphological criteria have less false positive diagnoses of malignant lesions than of kinetic criteria. ${ }^{19-21}$ This finding was comparable to the study of Goto et al., ${ }^{22}$ which concluded that the sensitivity and specificity of the morphologic pattern were significantly higher than those of the enhancement features based on a study of 144 malignant and 60 benign breast lesions. ${ }^{22}$

Other studies have shown that most (60-80\%) enhancing lesions recommended for biopsy are ultimately determined to be benign. ${ }^{23,24}$ Cystic apocrine metaplasia is a subset of fibrocystic changes featuring discrete clustered cysts that are lined by apocrine epithelium. Alexander et al. ${ }^{18}$ found that most (67\%) MRI-guided breast biopsies are performed on benign lesions, with many containing varying elements of fibrocystic change. ${ }^{18}$ Also, recent studies have described an increased detection of apocrine lesions with MRI.

In the short literature reviewed the most relevant features in MRI in the FCC is non-mass regional enhancement and predominantly curves type II. Nodular enhancements in general tend to have smooth edges and size no larger than $2 \mathrm{~cm}$. Yabuuchi $\mathrm{H}$ et al. ${ }^{25}$ found that lesions larger than $10 \mathrm{~mm}$ tended to be malignant, and lesions equal or smaller than $5 \mathrm{~mm}$ tended to be benign. Lesions with early enhancement peak tended to be malignant, whereas those with persistent enhancement tended to be benign. ${ }^{25}$

Also, Liberman L et al. ${ }^{26}$ studied the importance of lesion size on the positive predictive value (PPV) of biopsy in MRI detected breast lesions. It was found that the frequency of malignancy increased significantly with lesion size. Those lesions less than $5 \mathrm{~mm}$ had a $3 \%$ chance of being malignant, lesions with size $5-9 \mathrm{~mm}$ a $17 \%$ and lesions $10-14 \mathrm{~mm}$ a $25 \%$ malignancy rate. So they concluded that biopsy is rarely for lesions smaller than $5 \mathrm{~mm}$ because of their low (3\%) likelihood of cancer. ${ }^{26}$

Florea et al. ${ }^{27}$ establish that hypovascular lesions, non-rigid focal masthopatic changes are usually associated with non proliferative lesions. Stiff or hypervascular lesions better correlate with proliferative changes, and for those cases with imaging risk features, such as wash-out, differential diagnosis with in situ or invasive neoplasia is imposible on imaging criteria alone. ${ }^{27}$

Breast MRI compared to mammography and sonography, had worse diagnostic performance $(82 \%, 55 \% \mathrm{v}$ and $70 \%$ false positive rate, respectively). This fact actually supports the unreliability of using the pattern of enhancement kinetics in diagnosing breast malignancy. ${ }^{15}$

\section{Conclusion}

This entity is a real challenge for the breast radiologist. Why? , because more than $50 \%$ of lesions we see in the context of FCC show morphological and kinetic criteria of malignancy, so in the present moment we cannot avoid biopsies, much less when it comes to a patient with breast cancer. It's also necessary to know more about this disease to properly select patients which need more aggressive treatments.

I consider further studies are needed about the behavior of a simple and complex as the FCC in the field of breast MRI pathology.

\section{Acknowledgements}

None.

\section{Conflict of interest}

Author declares that there is no conflict of interest.

\section{References}

1. Gallo JL, Mas MP, Vico I, et al. Mastopatia fibroquistica. Aspectos controvertidos Fibrocystic breast disease. Controversial aspects. Clínica e Investigación en Ginecología y Obstetricia. 2013;40(6):269-276. 
2. Martin Etchart Lecciones de Anatomia Patológica. Procesos no tumorales de mama. Chile;

3. Love S, Gelman RS, Silen W. Fibrocystic "disease" of the breast, a non desease? The New England Journal of Medicine. 1982;307(16):1010-1014

4. Kopans D. Breast Imaging. 2nd ed. 1999. 18p.

5. Morris EA. Review of Brest MRI: indications and limitations. Semin Roentgenol. 2001;36(3):226-237.

6. Nunes LW, Schnall MD, Orel SG, et al. Correlation of lesion appearance and histologic findings for the nodes of a breast MR imaging interpretation model. Radiographics. 1999;19(1):79-92.

7. Guray M, Sahin A. Benign breast deseases: Clasification, diagnosis and management. Oncologist. 2006;11(5):435-449.

8. Fitzgibbons PL, Henson DE, Hutter RV. Benign breast changes and the risk for subsequent breast cancer: an update of the 1985 consensus statement. Cancer Committee of the College of American Pathologists. Arch Pathol Lab Med. 1998;122(12):1053-1055.

9. Sarnelli R, Squartini F. Fibrocystic condition and "at risk" lesions in asymptomatic breasts: a morphologic study of postmenopausal women. Clin Exp Obstet Gynecol. 1991;18(4):271-279.

10. Wu C, Ray RM, Lin MG, et al. A case-control study of risk factors for fibrocystic breast conditions. Am J Epidemiol. 2004;160(10):945-960.

11. Santen RJ, Mansel R. Benign breast disorders. The New England Journal of Medicine. 2005;353(3):275-285.

12. Morris E, Liberman L. Breast MRI: diagnosis and intervention. Radiology. 2010;243(1):117.

13. Kiyak G, Asik E, Yazgan A. Importance of magnetic resonance imaging in patients with fibrocystic changes. Bratisl Lek Listy. 2011;112(9):506-509.

14. Scott EB. Fibrocystic breast disease. Am Fam Physician. 1987;36:119-126.

15. Chen JH, Nalcioglu O, Su MY. Fibrocystic change of the breast presenting as a focal lesión mimicking breast cáncer in MR imaging. $J$ Magn Reson Imaging. 2008;28(6):1499-1505.

16. Zhu JZ, Kurihara Y, Kanemaki Y, et al. Diagnostic accuracy of highresolution MRI using a microscopy coil for patients with presumed DCIS following mammography screening. J Magn Reson Imaging 2007;25(1):96-103.
17. Teifke A, Behr O, Schmidt M, et al. Dynamic MR imaging of breast lesions: correlation with microvessel distribution pattern and histologic characteristics of prognosis. Radiology. 2006;239(2):351-360.

18. Alexander DR, Marotti J, Bond J, et al. Breast MRI-Detected Cystic Apocrine Metaplasia: Imaging Features With Microvessel Analysis and Histologic Correlation. AJR Am J Roentgenol. 2015;204(1):211-218.

19. Van den Bosch MA, Daniel BL, Mariano MN, et al. Magnetic resonance imaging characteristics fibrocystic change of the breast. Invest Radiol. $2005 ; 40(7): 436-441$.

20. Chen JH, Liu H, Baek HM, et al. MR Imaging Features of Fibrocystic Change of the Breast. Magn Reson Imaging. 2008;26(9):1207-1214.

21. Binokay F, Soyupak S, Bicakci K, et al. Wide spectrum of fibrocystic changes: MR findings. ECR. 2010.

22. Goto M, Ito H, Akazawa K, et al. Diagnosis of breast tumors by contrastenhanced MR imaging: comparison between the diagnostic performance of dynamic enhancement patterns and morphologic features. J Magn Reson Imaging. 2007;25(1):104-112.

23. Siziopikou KP, Jokich P, Cobleigh M. Pathologic findings in MRI-guided needle core biopsies of the breast in patients with newly diagnosed breast cancer. Int J Breast Cancer. 2011;2011:613285.

24. Schell AM, Rosenkranz K, Lewis PJ. Role of breast MRI in the preoperative evaluation of patients with newly diagnosed breast cancer. $A J R A m$ J Roentgenol. 2009;192(5):1438-1444.

25. Yabuuchi H, Kuroiwa T, Kusumoto C, et al. Incidentally detected lesions on contrast-enhanced MR imaging in candidates for breast-conserving therapy: correlation between MR findings and histological diagnosis. $J$ Magn Reson Imaging. 2006;23(4):486-492.

26. Liberman L, Mason G, Morris EA, et al. Does size matter? Positive predictive value of MRI-detected breast lesions as a function of lesion size. AJR Am J Roentgenol. 2006;186(2):426-430.

27. Florea M, Chiorean AR, Roman RA, et al. Imaging spectrum of breas focal fibrocystic changes: mammography, conventional ultrasound, elastography and MRI appearances; pathology correlations and differential diagnosis. ECR. 2014. 